\title{
Genome-wide DNA methylation and hydroxymethylation analysis reveal human menstrual blood-derived stem cells inhibit hepatocellular carcinoma growth through oncogenic pathway suppression via regulating $5-\mathrm{hmC}$ in enhancer elements
}

\author{
Yichen Wu, Xin Chen, Yongjia Zhao, Yanling Wang, Yifei Li and Charlie Xiang*
}

\begin{abstract}
Background: Epigenetic alteration is an important indicator of crosstalk between cancer cells and surrounding microenvironment components including mesenchymal stem cells (MSC). Human menstrual blood-derived stem cells (MenSCs) are novel source of MSCs which exert suppressive effects on cancers via multiple components of microenvironmental paracrine signaling. However, whether MenSCs play a crucial role in the epigenetic regulation of cancer cells remains unknown.

Methods: Epigenetic alterations of hepatocellular carcinoma (HCC) mediated by MenSCs were examined by immunofluorescence, ELISA, and RT-PCR assays. The suppressive impact of MenSCs on HCC was investigated in vitro using CCK8, apoptosis, wound healing, and invasion assays and in vivo using a xenograft mice model. MeDIPseq, hMeDIP-seq, and RNA-seq were used to identify the genome-wide pattern of DNA methylation and hydroxymethylation in HCC cells after MenSC therapy.

Results: We show that HCC cells display distinct genome-wide alterations in DNA hydroxymethylation and methylation after MenSC therapy. MenSCs exert an inhibitory effect on HCC growth via regulating 5-hmC and 5-mC abundance in the regulatory regions of oncogenic pathways including PI3K/AKT and MAPK signaling, especially in enhancers and promoters. FOXO3 expression is rescued via reversal of 5-hmC and 5-mC levels in its enhancers and contributes to the activation of downstream apoptosis. Inactivation of the MAPK pathway further disrupts c-myc-mediated epithelialmesenchymal transitions (EMT). Additionally, chemotherapy resistance-associated genes including ID4 and HMGA1 are suppressed via amending 5-hmC and 5-mC abundance at their regulatory regions. HMGA1 and BYSL might be potential targets for gene-modified MSC therapy.
\end{abstract}

Conclusions: Our results confirm that MSCs could regulate the epigenetic mechanism of HCC cells and provide a novel concept for a modified MSC strategy or combination therapy with chemotherapeutics based on epigenetics.

Keywords: Mesenchymal stem cell, HCC, Epigenetics, 5-Hydroxymethylcytosine, Enhancer

\footnotetext{
* Correspondence: cxiang@zju.edu.cn

State Key Laboratory for Diagnosis and Treatment of Infectious Diseases, Collaborative Innovation Center for Diagnosis and Treatment of Infectious Diseases, The First Affiliated Hospital, College of Medicine, Zhejiang University, Hangzhou, China
}

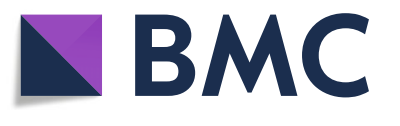

(c) The Author(s). 2019 Open Access This article is distributed under the terms of the Creative Commons Attribution 4.0 International License (http://creativecommons.org/licenses/by/4.0/), which permits unrestricted use, distribution, and reproduction in any medium, provided you give appropriate credit to the original author(s) and the source, provide a link to the Creative Commons license, and indicate if changes were made. The Creative Commons Public Domain Dedication waiver (http://creativecommons.org/publicdomain/zero/1.0/) applies to the data made available in this article, unless otherwise stated. 


\section{Background}

Tumor malignant transformation is driven by a long-term gradual accumulation of genetic insults and epigenetic abnormalities $[1,2]$. The dynamic plasticity of the epigenome in cancers closely correlates to microenvironmental cues, including infection [3], chronic inflammation [4], and hypoxia [5]. DNA methylation is one of the widely studied epigenetic modifications and plays an important role in diverse cellular processes, including genomic imprinting, embryonic development, and tumorigenesis [6-8]. The balance of DNA methylation in the genome is principally maintained by tet methylcytosine dioxygenases (TETs), which mediate demethylation, and DNA methyltransferases (DNMTs), which mediate methylation [9]. The stepwise accumulation of a DNA methylation burden in promoter CpG islands has been conclusively demonstrated to contribute to suppressor inactivation [10]. Active demethylation in mammals is mediated by the TET family, including TET1, TET2, and TET3, which oxidize 5-mC to 5-hydroxymethylcytosine $(5-\mathrm{hmC})$, and further to 5-formylcytosine (5-fC) and 5-carboxylcytosine (5-caC) [11]. 5 -hmC, a vital demethylation intermediate, is frequently diminished in tumorigenesis. Because $5-\mathrm{hmC}$ is enriched in regulatory regions, changes in its distribution usually involve variation in transcriptional expression [12]. In addition, 5-hmC abundance in the genome adjusts dynamically in response to altered environmental stimuli $[13,14]$. Hence, 5-hmC has been recommended as a sensitive marker for indicating cell burden $[15,16]$.

MSCs are adult pluripotent progenitor cells of multiple mesenchymal lineages that are widely studied in degenerative and inflammatory disorders [17]. Given their inflammation- and tumor-homing properties, MSCs have been considered as potential vehicles for cancer therapy that are able to target the sites of even microscopic tumors. Recently, strategies involving gene-modified MSCs that carried TRAIL [18], IL-12 [19], and an oncolytic virus [20] have been developed to improve their therapeutic effects. However, the clinical feasibility of MSCs for cancer therapy is hampered by a lack of sufficient understanding of the mechanisms of interaction between MSCs and cancer cells. Hence, a great deal of research is still needed to explore the role of MSCs in the regulation of cancer microenvironments. MenSCs, a novel type of MSCs, exhibit high proliferation, hypoimmunogenicity, and multiple differentiation potential [21, 22]. Unlike the MSCs isolated from the bone marrow, adipose, and umbilical cord, MenSCs are very easily obtained, being isolated from human menstrual fluid without pain, invasive procedures, or ethical issues. Previous studies have demonstrated the potential of MenSC-based therapy in different disease models, including liver fibrosis, fulminant hepatic failure, and acute lung injury [23-25]. As a cancer therapy, MenSCs have been shown to block the growth of ovarian cancer [26]. However, the potential of MenSCs to act on other cancers remains unknown.

Hepatocellular carcinoma (HCC) is one of the cancers in which pathogenic processes intimately correlate with epigenetic dysregulation under long-term burdens of infection and chronic inflammation. Previously, gene-modified MSCs applied to acute, chronic liver disease and even precancerous lesions have been shown to have a significantly beneficial effect. However, the role of MSCs in the epigenetic regulation of $\mathrm{HCC}$ is still unknown.

In the present study, we aimed to investigate whether MenSCs affect epigenetic regulation in HCC and exert anticancer effects via specific epigenetic alterations. Based on the multi-platform integration, we provided important evidence of distinct 5-hmC and 5-mC landscapes in HCC cells after MenSC therapy. Then, we showed that MenSCs exerted an anticancer effect via suppressing oncogenic pathways and reviving tumor suppressors through altering the abundance and distribution of $5-\mathrm{hmC}$ and $5-\mathrm{mC}$ in regulatory regions, especially enhancers. Our study not only contributes to demonstrate the epigenetic mechanism between HCC and microenvironment, but also suggests MenSCs would be promising vehicles for a modified MSC strategy based on epigenetics or combination therapy with chemotherapeutics in HCC.

\section{Materials and methods}

\section{Cell culture}

Menstrual blood was obtained from healthy female volunteers after informed consent, and MenSC isolation was approved by the Ethics Committee of Zhejiang University. MenSCs were isolated as previously described [27]. Briefly, menstrual blood samples were collected with DivaCups (Kitchener, ON, Canada) from volunteers during menstruation. The mononuclear cells were separated via density gradient centrifugation with Ficoll-Paque (Fisher Scientific, USA). The interlayer cells were collected carefully and cultured in Chang Medium (S-Evans Biosciences, Hangzhou, China). After 48 h, the MenSCs grew by adhering to the wall and nonadherent hematopoietic cells were discarded. The MenSCs used in experiments were at 3rd to 8th passage. Human liver cancer cell lines HepG2 and $\mathrm{HuH}-7$ and human fibroblast MRC-5 were obtained from the National Infrastructure of Cell Line Resources (China). HCC cells and MRC-5 cells were cultured in MEM GlutaMAX (Gibco, USA) with 10\% FBS under a $5 \% \mathrm{CO}_{2}$ atmosphere.

Transwell chamber system with a $0.4-\mu \mathrm{m}$ pore size was utilized to construct the cell coculture model. To identify the specific impact of MenSCs on HCC cells, we considered HCC cells cocultured with MRC-5 cells or with medium only served as control groups. HCC cells were seeded into the lower chamber, and MenSCs or MRC-5 cells were seeded into the upper chamber in the same 
medium. HCC cells were collected after coculturing with MenSCs or MRC- 5 cells for 24,48 , or $72 \mathrm{~h}$, respectively.

\section{Methylated DNA immune-precipitation sequencing (MeDIP-Seq) and hydroxymethylated DNA immunoprecipitation sequencing (hMeDIP-Seq)}

Purified genomic DNA was sonicated to obtain fragments with a size range of 200-800 bp for hMeDIP-seq or 200$500 \mathrm{bp}$ for MeDIP-seq. Following end repair of the DNA samples with the addition of deoxyadenosine and adaptor ligation based on the Illumina paired-end protocol, the DNA fragments were immunoprecipitated with anti-5hydroxymethylcytosine or anti-5-methylcytosine antibody. Then, the precipitated fragments were amplified by PCR, and 300-900-bp DNA fragments were selected by using AMPure XP beads. The completed libraries were denatured with $0.1 \mathrm{M} \mathrm{NaOH}$ to generate single-stranded DNA molecules, captured on an Illumina flow cell, and amplified in situ. The libraries were then sequenced on the Illumina HiSeq 4000 following the HiSeq 3000/4000 SBS Kit (300 cycles) protocol. After base calling, low-quality reads were passed, and the clean reads were aligned to the UCSC human reference genome hg19 using HISAT2 software (V2.1.0).

\section{RNA-Seq}

Total RNA was isolated from cancer cells by using the AllPrep ${ }^{\circledR}$ DNA/RNA Mini Kit (Qiagen, Germany) according to the manufacturer's instructions. After assessment of the quantity, quality, and integrity of RNA, the mRNA was enriched with Oligo (dT) beads and rRNA was removed using the Ribo-Zero ${ }^{\mathrm{Tm}}$ Magnetic Gold Kit (Epicentre, USA). The mRNA fragment library was prepared using the KAPA Stranded RNA-Seq Library Prep Kit (Illumina, USA) according to the manufacturer's instructions. After mRNA amplification using qPCR, library quality was assessed using the Agilent 2100 Bioanalyzer. The mRNA fragment library was sequenced on the Illumina HiSeq 4000.

\section{RT-PCR}

A total of 100-500 ng RNA was used to synthesize cDNA with the PrimeScript ${ }^{\mathrm{TM}}$ RT Reagent Kit (Takara, Japan). The mRNA expression levels of TET1/2/3 and DNMT1/ $3 \mathrm{~A} / 3 \mathrm{~B}$ were analyzed using $\mathrm{SYBR}^{\odot}$ Premix Ex $\mathrm{Taq}^{\mathrm{Tm}}$ (Takara, Japan) by real-time PCR. The $\beta$-actin gene was used as an internal reference. The sequences of primers for RT-PCR are shown in (Additional file 1: Table S1). Relative changes in gene expression were calculated according to the $2^{-\Delta \Delta C T}$ method.

\section{Immunohistochemical analysis}

Immunohistochemical analysis was performed as described in our previous study. Briefly, all tumor xenograft sections were deparaffinized with xylene and rehydrated through an alcohol gradient, and then, endogenous peroxidase was inactivated by $0.5 \% \mathrm{H}_{2} \mathrm{O}_{2}$ for $10 \mathrm{~min}$. Subsequently, the sections were blocked with $5 \%$ normal goat serum for $1 \mathrm{~h}$ and washed three times with PBS, followed by incubation at $4{ }^{\circ} \mathrm{C}$ overnight with one of the following primary antibodies: anti-5-hmC (1:100, Active Motif), anti-5mC (1:100, Active Motif), anti-TET1 (1:300, Abcam), anti-TET2 (1:300, Abcam), anti-TET3 (1:300, Abcam), anti-DNMT1 (1:100, Abcam), anti-DNMT3A (1:500, Abcam), antiDNMT3B (1:100, Abcam), anti-Ki 67 (1:200, Abcam), and anti-luciferase (1:1000, Abcam). The sections were incubated with horseradish peroxidase (HRP)-conjugated secondary antibody at room temperature for $1 \mathrm{~h}$ and developed with a DAB kit.

\section{Immunofluorescence staining}

HCC cells were fixed with 4\% paraformaldehyde for 15 min and permeabilized with 0.1 Triton X-100 in PBS for 20 min. To denature the DNA, the cells were treated with $4 \mathrm{M} \mathrm{HCl}$ for $15 \mathrm{~min}$ at room temperature and neutralized with $100 \mathrm{mM}$ Tris- $\mathrm{HCl}$ (pH 8.5) for $10 \mathrm{~min}$. After washing with PBS, the cells were blocked with 5\% BSA for $1 \mathrm{~h}$. Then, the cells were incubated with primary antibodies overnight at $4{ }^{\circ} \mathrm{C}$. After washing with PBS, the cells were incubated with secondary antibody (Alexa Fluor 488 goat anti-rabbit IgG Abcam; Alexa Fluor 633 goat anti-mouse IgG, Thermo Fisher) and then stained with DAPI. The plates were scanned, and images were obtained with an Olympus IX83 microscope.

\section{Western blotting}

HCC cells were lysed in ice-cold RIPA lysis buffer with protease inhibitor and phosphatase inhibitor. Protein concentration was determined by using the BCA method. Thirty micrograms of total protein was separated by SDS-PAGE and transferred onto PVDF membranes (Millipore, USA). The membranes were blocked in $0.5 \%$ BSA for $1 \mathrm{~h}$ at room temperature and incubated overnight with antibodies to PTEN, FOXO3, PI3K, AKT, p-AKT, ERK1/2, p-ERK1/2, C-myc, active caspase3, Bcl2, Bax, E-cadherin, TIMP1, MMP2, snail/slug, twist, and GAPDH (Abcam, USA). Then, the membranes were incubated with the corresponding HRP-labeled secondary antibodies for $1 \mathrm{~h}$ at room temperature. Chemiluminescent signals were captured by the Imager (Tiangen, China).

\section{Animal work}

All animal experiments were approved by the Ethics Committee of the First Affiliated Hospital, Zhejiang University. Four-week Balb/c nude mice were obtained from Slac Laboratory Animal Corporation (Shanghai, China). To generate the xenograft model, $2 \times 10^{6}$ HepG 2 cells in $100-\mu l$ serum-free medium were implanted subcutaneously into 
10 mice at the right foreleg. When the diameter of the tumor under the skin reached $5 \mathrm{~mm}$ (about 21 days), 10 mice were randomly divided into two groups $(n=5$ per group). The MenSC therapy group was treated with tail vein administration of $5 \times 10^{5}$ MenSCs labeled with luciferase in $400-\mu \mathrm{l}$ serum-free medium every 6 days for three successive times, and the control group received 400- $\mu \mathrm{l}$ serum-free medium only. After first tail vein administration, the tumor volume of each mouse was calculated as (length $\times$ width $\times$ width) $/ 2$ every 3 days until the mice were sacrificed. To evaluate the tumor-tropism of MenSCs, bioluminescence optical imaging was obtained 3 days after the last MenSC treatment. Mice were sacrificed after the bioluminescence optical imaging (about 36 days after HepG 2 implantation), and the xenograft tumor was collected for immunohistochemical analysis.

\section{Flow cytometry analysis for surface markers of MenSCs and apoptosis of HCC cells}

After collection and washing twice with stain buffer (BD Biosciences, CA, USA), MenSCs were incubated with antibodies of surface markers including CD29, CD34, CD45, CD73, CD90, CD105, CD117, and HLA-DR (Becton Dickinson, NJ, USA) for $20 \mathrm{~min}$. The stained cells were washed twice with stain buffer and resuspended in $500 \mu \mathrm{l}$ of stain buffer to be analyzed by FC500 flow cytometer (Beckman Coulter, CA, USA).

HCC cells were seeded in six-well plates and cocultured with MenSCs, MRC-5, and medium only for $72 \mathrm{~h}$, respectively. Cell apoptosis was detected using Annexin V/PI detection kit (BD Biosciences, CA, USA) by FC500 flow cytometer. Data were analyzed using Flowjo software (Tree Star, OR, USA).

\section{Cell viability assay}

HCC cells and MenSCs were seeded into the 24-well plates and upper transwell chambers, respectively. Cell viability was assessed by Cell Counting Kit-8 (Dojindo) according to the manufacturer's protocol.

\section{Clonogenic assay}

HCC cells were seeded in six-well plates at a density of 1000 cells per well and cocultured with MenSCs, MRC-5, and medium only using transwell coculture system for 7 days. After incubation, HCC cells were fixed with $4 \%$ paraformaldehyde and stained with $0.1 \%(w / v)$ crystal violet. Megascopic cell colonies were calculated under an Olympus IX83 microscope, and each measurement was performed in triplicate.

\section{5-hmC ELISA}

For measuring the global DNA hydroxymethylation levels, we used MethylFlash ${ }^{\mathrm{Tm}}$ Global DNA hydroxymethylation (5-hmC) ELISA Easy Kit (Epigentek) to detect the 5-hmC level in HCC cells after MenSC treatment. ELISA was performed according to the manufacturer's protocol.

\section{Wound healing assay}

HCC cells were seeded in IBIDI wound healing system in 12-well plates. After cells reached a confluence of more than $90 \%$, removing the IBIDI stands to form a straight line without cells in the middle of the wells. Then, HCC cells were cocultured with MenSCs, MRC-5, and medium only, respectively. Photographs were taken from 0 to $48 \mathrm{~h}$ on an optical microscope.

\section{Transwell invasion assay}

The upper chambers with $8-\mu \mathrm{m}$ pore size were precoated with $1 \mathrm{mg} / \mathrm{ml}$ Matrigel (BD Biosciences, USA) for $1 \mathrm{~h} .2 \times$ $10^{4}$ of HCC cells in 100- $\mu$ l medium were added into upper chambers, and $2 \times 10^{4}$ of MenSCs and MRC- 5 cells were seeded in the lower chamber beforehand. After 48-h incubation, HCC cells were fixed with $4 \%$ paraformaldehyde and stained with $0.1 \%$ crystal violet. Stained cells were observed and counted under a microscope.

\section{Statistical analysis}

All data analysis was conducted using SPSS 18.0 statistical software and GraphPad Prism 6 software. All values were expressed as the means \pm SD. Student's $t$ test was used for determining significant differences between groups. Two-way ANOVA was used to analyze CCK8 assay. The difference of two groups from five time points in CCK8 assay was performed by Sidak's multiple comparisons test. Functional enrichment analysis was performed by pathway analysis using the DAVID web server (http://david.abcc.ncifcrf.gov/). Genes with DMRs and DHMRs in enhancers were mapped to their respective human orthologs, and the lists were submitted to DAVID for enrichment analysis to determine significant KFGG-pathway categories. For time-to-event analyses, survival estimates were calculated by the Kaplan-Meier analysis, and groups were compared with the log-rank test. $P<0.05$ was considered statistically significant.

\section{Results \\ MenSCs had an anticancer effect associated with dynamic DNA methylation alteration}

Flow cytometry was performed to identify the phenotype of MenSCs, and the results showed that MenSCs were positive for $\mathrm{CD} 29, \mathrm{CD} 73, \mathrm{CD} 90$, and $\mathrm{CD} 105$ and negative for CD34, CD45, CD117, and human leukocyte antigen DR (HLA-DR) (Fig. 1a). Moreover, MenSCs were able to differentiate into adipocytes, chondroblasts, and osteoblasts (Fig. 1b). Thus, MenSCs had low immunogenicity and multiple differentiation potential and were suitable for transplantation into animals or humans. 

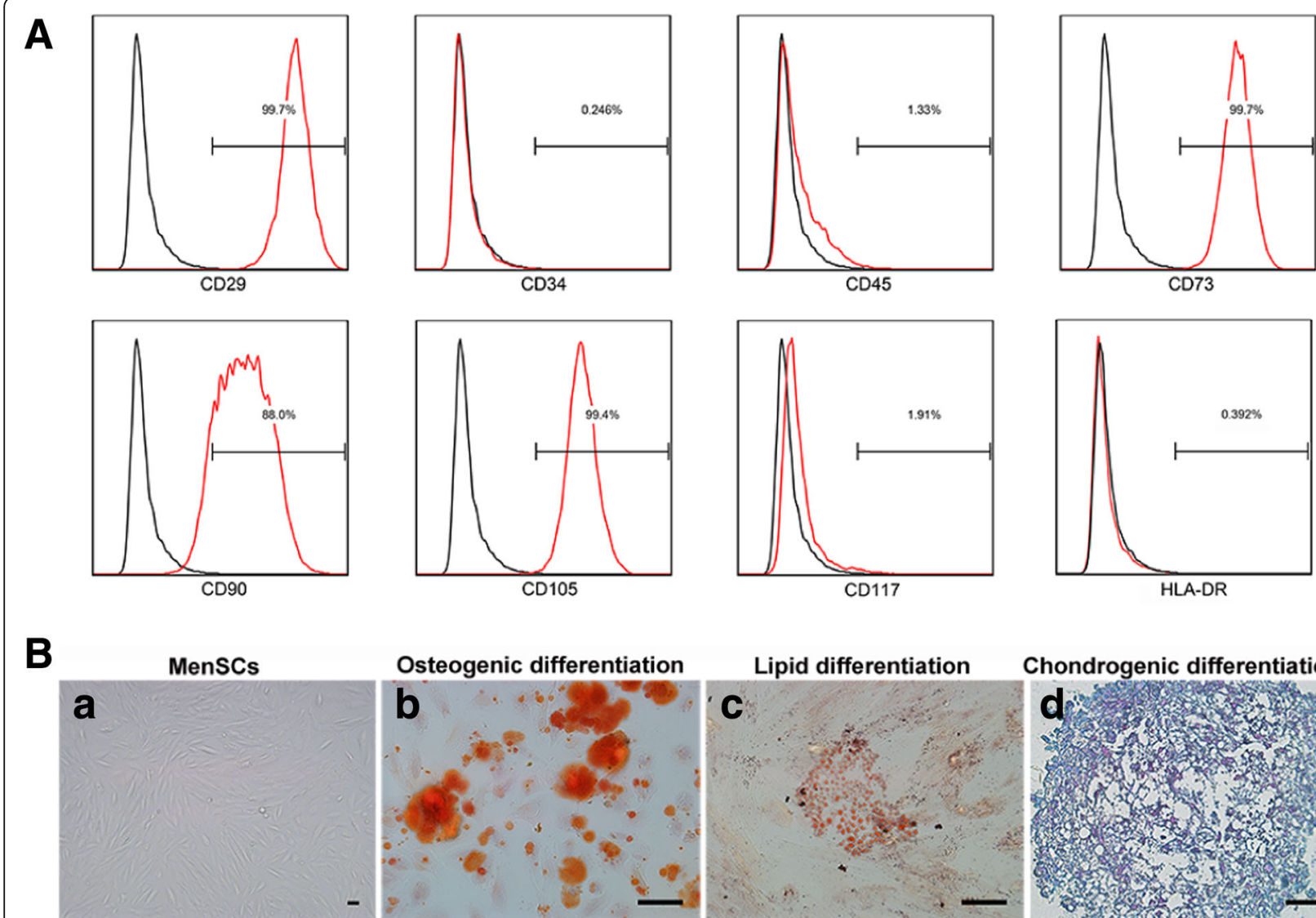

Osteogenic differentiation

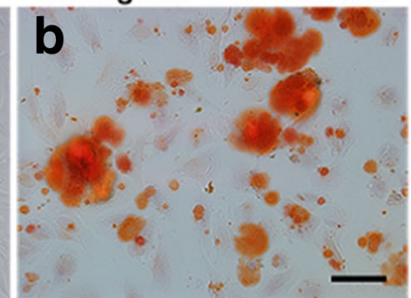

\section{Lipid differentiation}
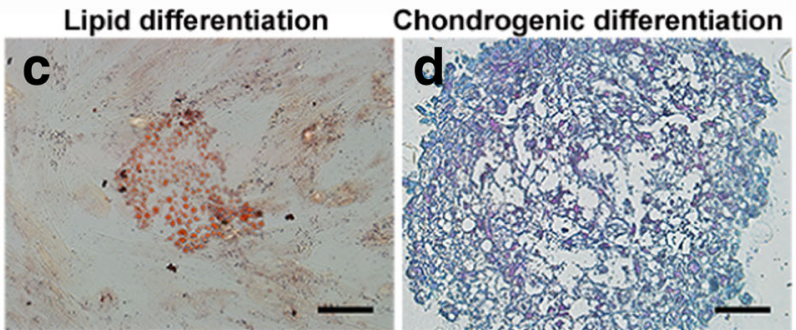

Fig. 1 Characterization of MenSCs. a Flow cytometry analysis showed surface markers of MenSCs. b Representative images showed morphology and tri-lineage differentiation potential of MenSCs. Scale bar $50 \mu \mathrm{m}$

To investigate whether MenSCs play a role in the epigenetic regulation of $\mathrm{HCC}$ cells, we measured the 5-hmC level of HCC cells after MenSC coculture for 72 $\mathrm{h}$ using immunofluorescence (IF) assay and ELISA assay. IF assay showed that 5 -hmC level in HCC cells was significantly increased after MenSC coculture (Fig. 2a). ELISA assay further showed the 5-hmC level of HCC with MenSC coculture was significantly increased than that of the control group (HepG2 $0.0288 \pm 0.0033$ vs $0.0047 \pm 0.0022, P=0.0038 ; \mathrm{HuH}-70.0248 \pm 0.0049$ vs $0.0085 \pm 0.0024, P=0.0415$, Fig. $2 b)$. Then, we detected DNA methylation and demethylation enzymes, including TETs and DNMTs in HCC cells, through a real-time PCR assay. Results showed that TET1 expression in HepG2 was gradually enhanced in a time-dependent manner from 24 to $72 \mathrm{~h}$ during MenSC coculture. However, TET3 expression and that of the DNMTs, especially DNMT1, gradually decreased (Fig. 2c). Additionally, TET1 and TET2 expression was clearly enhanced, while DNMTs did not appear to decrease evidently in $\mathrm{HuH} 7$ under MenSC coculture (Fig. 2c). These results suggested that MenSCs dynamically regulate DNA methylation in diverse HCC cells via different methylation enzymes. Next, we measured the biological effect of MenSCs on HCC growth and evaluated whether the epigenetic alterations mediated by MenSCs were associated with cell growth. IF assay showed that Ki67 levels in HepG2 and HuH-7 were obviously decreased after MenSCs coculture for $72 \mathrm{~h}$ (Fig. 2d). Furthermore, CCK8 assays showed that, compared with the controls, MenSCs significantly suppressed the proliferation of HCC cells (Fig. 2e). However, MRC-5 clearly promoted the proliferation of $\mathrm{HCC}$ cells, indicating that fibroblast cells could fuel cell growth in the tumor microenvironment. Additionally, MenSCs inhibited the clonogenic potential of HepG2 and HuH-7 (Fig. 2f). Compared with control groups, the clone number of the MenSC group was significantly decreased. These results indicated that MenSCs had a suppressive impact on the growth of HCC cells in vitro. Moreover, the suppressive effect of MenSCs on the HCC growth was associated with epigenetic alterations.

Furthermore, we wanted to validate whether MenSCs play a role in epigenetic regulation of HCC in vivo. As 


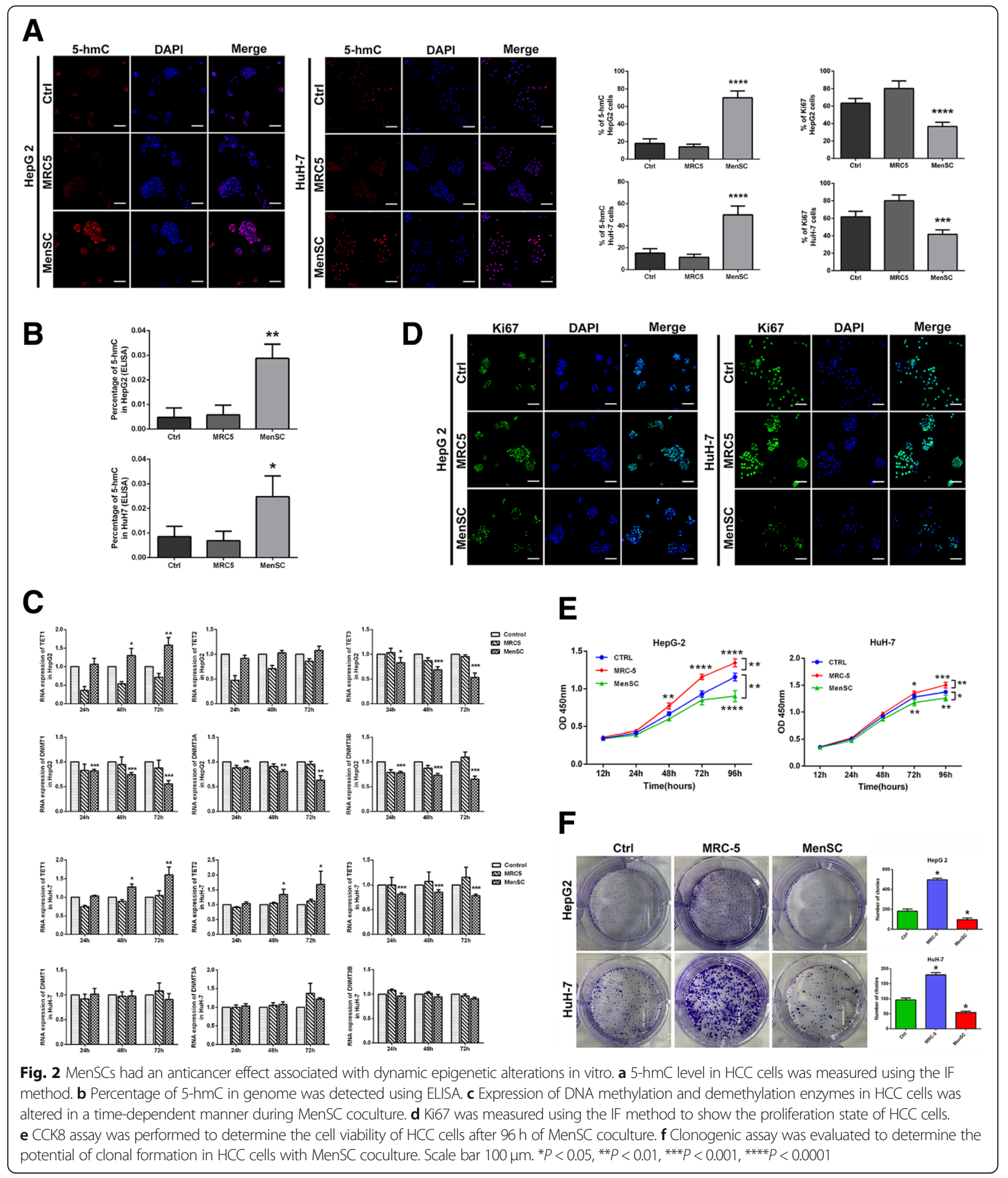

shown in Fig. 3a and b, MenSCs that carried luciferase could specifically target the tumor site and we also used the antibody of anti-luciferase to track the distribution of MenSCs in HCC tissues. Tumor volume and weight of mice injected with MenSCs were significantly lower than those of the control group after three times of tail vein injection (Fig. 3c, d). IHC assay showed the 5-hmC level in $\mathrm{HCC}$ with MenSC injection was significantly higher than that in the control group (Fig. 3e). Moreover, the expression of TET1 and TET2 was clearly 


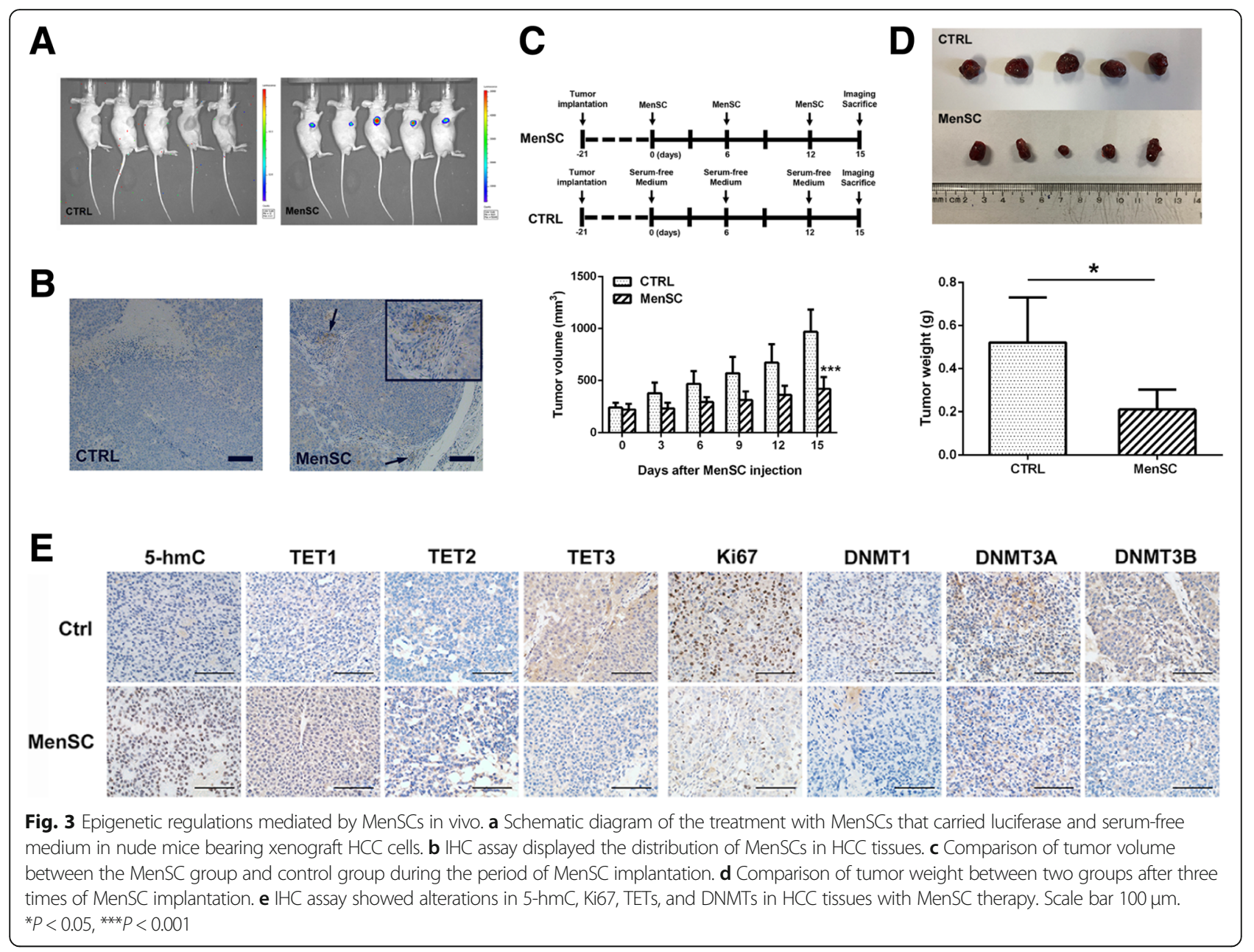

increased, and the expression of TET3, DNMT1 DNMT3A, DNMT3B, and Ki67 were sharply reduced in HCC with MenSC injection. Collectively, these results demonstrated that MenSCs could suppress the growth of $\mathrm{HCC}$ and modify epigenetic regulation in vivo.

\section{MenSCs influenced global patterns of hydroxymethylome and methylome in HCC}

Next, we used the hMeDIP-seq and MeDIP-seq technologies to meticulously analyze whole-genome changes in methylome and hydroxymethylome of HepG2 after $72 \mathrm{~h}$ of MenSC coculture. We mapped the distribution of all hydroxymethylated and methylated signals in HCC cells (Fig. 4a). In intergenic regions, the proportion of 5-hmC signal was more abundant than that of the $5-\mathrm{mC}$. However, in promoter and genebody regions, 5 - $\mathrm{mC}$ level was more affluent than 5 -hmC level. Moreover, the alterations of 5 -hmC abundance tended to occur in promoter and intergenic regions after MenSC coculture, while 5-mC changes mainly happened in promoter regions (Fig. 4a). By graphing 5-hmC and 5-mC distribution across gene regions, we showed that $5-\mathrm{hmC}$ and 5-mC formed a deep dip around transcription start sites (TSSs) (Fig. 4b). Moreover, 5-mC formed a large peak to the right of TSSs. Both 5-mC and 5-hmC exhibited several small peaks along gene bodies toward the 3' gene terminus. Additionally, the abundance and distribution of 5-hmC and 5-mC in HCC cells exhibited significant alterations after MenSC coculture. These results suggested that changes of $5-\mathrm{hmC}$ and $5-\mathrm{mC}$ in different regions mediated by MenSCs may play different roles in transcription regulation.

Next, we mapped the distribution of differentially hydroxymethylated regions (DHMRs) and differentially methylated regions (DMRs) in all chromosomes. We found that alterations in 5-hmC and 5-mC occurred throughout the genome after MenSC treatment. Moreover, DHMRs and DMRs generally did not overlap in chromosomes (Fig. 4c). Furthermore, we aimed to explore which pathways were significantly differentially regulated in conjunction with MenSC-mediated epigenetic alterations. Interestingly, we found genes which have DHMRs or DMRs in enhancers were primarily associated with MAPK pathway, FOXO pathway, PI3K/Akt pathway, focal adhesion, endocytosis, and apoptosis (Fig. 4d, Additional file 1: Table S2 and S3). 


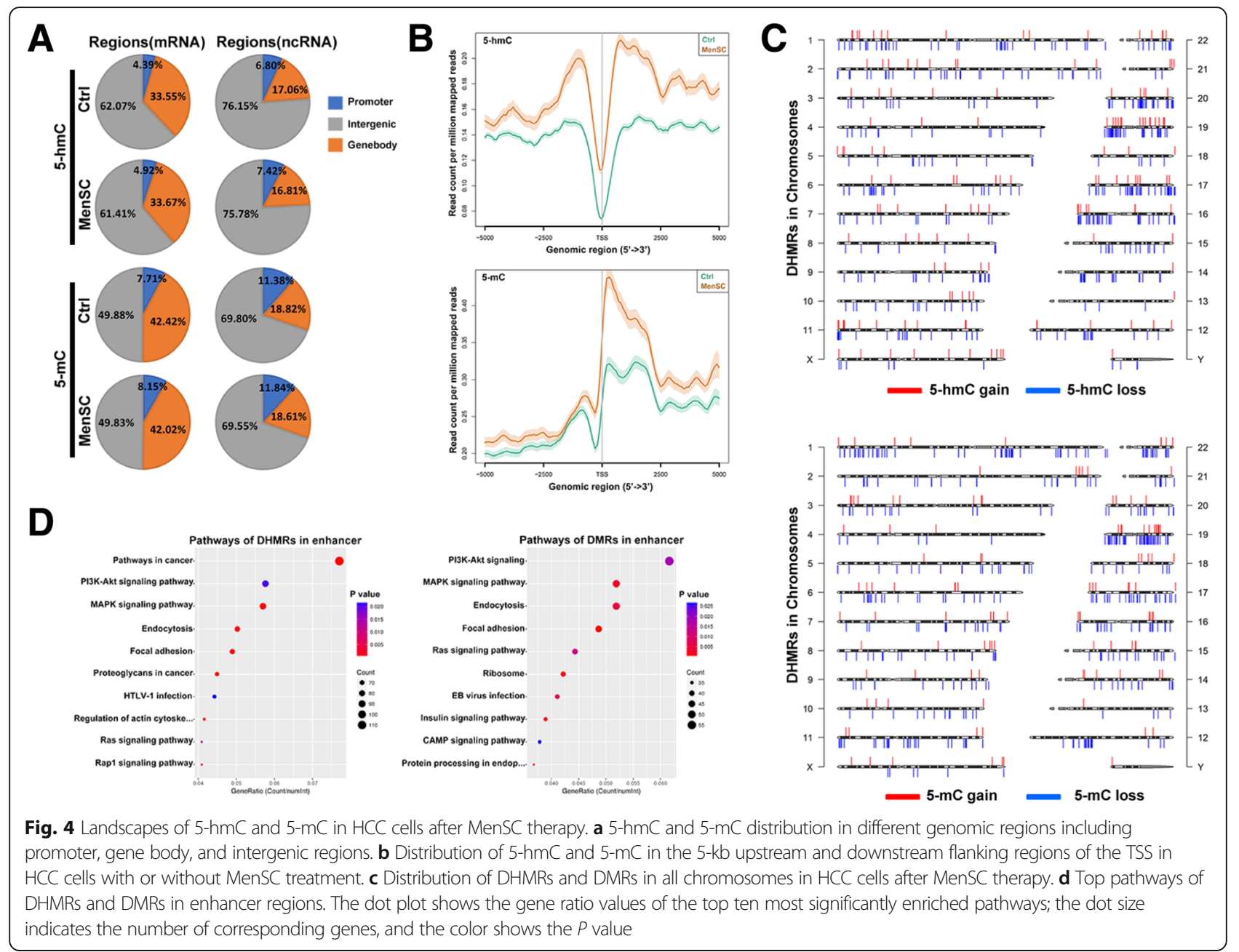

These results suggested that MenSCs might secrete various components to induce the activation of endocytosis of $\mathrm{HCC}$ cells and influence the microenvironment to regulate multiple biological functions including apoptosis and metastasis via epigenetic alterations.

\section{MenSCs promote HCC cell apoptosis through the PI3K/ AKT/FOXO pathway via regulating enhancer $5-\mathrm{hmC}$ and 5-mC levels}

Given that pathway analysis presented hydroxymethylated and methylated alterations were closely associated with PI3K/AKT pathway, we next verified which genes were regulated by epigenetic alterations. PTEN is the key negative regulatory factor of the PI3K/AKT pathway, and we found that the 5 -hmC level in enhancer region of PTEN (chr10:89989801-89990380) was significantly increased $(P=6.06 \mathrm{E}-07)$, while the 5 - $\mathrm{mC}$ level in the enhancer (chr10:90019261-90019640) was decreased following MenSC coculture $(P=2.07 \mathrm{E}-09$; Fig. 5a). However, the 5 -hmC and 5-mC abundances in enhancer regions of Akt1 $(P=3.31 \mathrm{E}-5)$ and Akt2 $(P=5.21 \mathrm{E}-5)$ were apparently altered in response to MenSC treatment. Moreover, MenSCs induced a reduction in $5-\mathrm{hmC}$ in the enhancer region of PIK3CD $(P=2.18 \mathrm{E}-05)$ and an increase in $5-\mathrm{mC}$ in the enhancer region of PIK3R2 $(P=$ 1.09E-05) to suppress the PI3K-mediated activation of Akt (Additional file 2: Figure S1). In addition, the FOXO pathway is one of the downstream targets of Akt and involves in the cell apoptosis. The $5-\mathrm{hmC}$ abundance in the enhancer of FOXO3 was also upregulated (chr6:108929921-108930200; $P=2.53 \mathrm{E}-6$; Fig. 5a). Additionally, promoter regions of apoptosis-related genes, including TNFRSF1A and Caspase7, showed significant increases in 5-hmC abundance (Additional file 2: Figure $\mathrm{S} 1)$. To explore whether alterations in $5-\mathrm{hmC}$ and $5-\mathrm{mC}$ in these regions influenced gene expression, we determined RNA and protein expression in HCC cells. Results showed that MenSCs could significantly upregulate PTEN and $\mathrm{FOXO} 3$ expression compared with control groups (Fig. 5b, c). PI3K, Akt, and phosphorylated Akt proteins were sharply decreased under coculture with MenSCs. Furthermore, downstream apoptotic proteins Bax and 


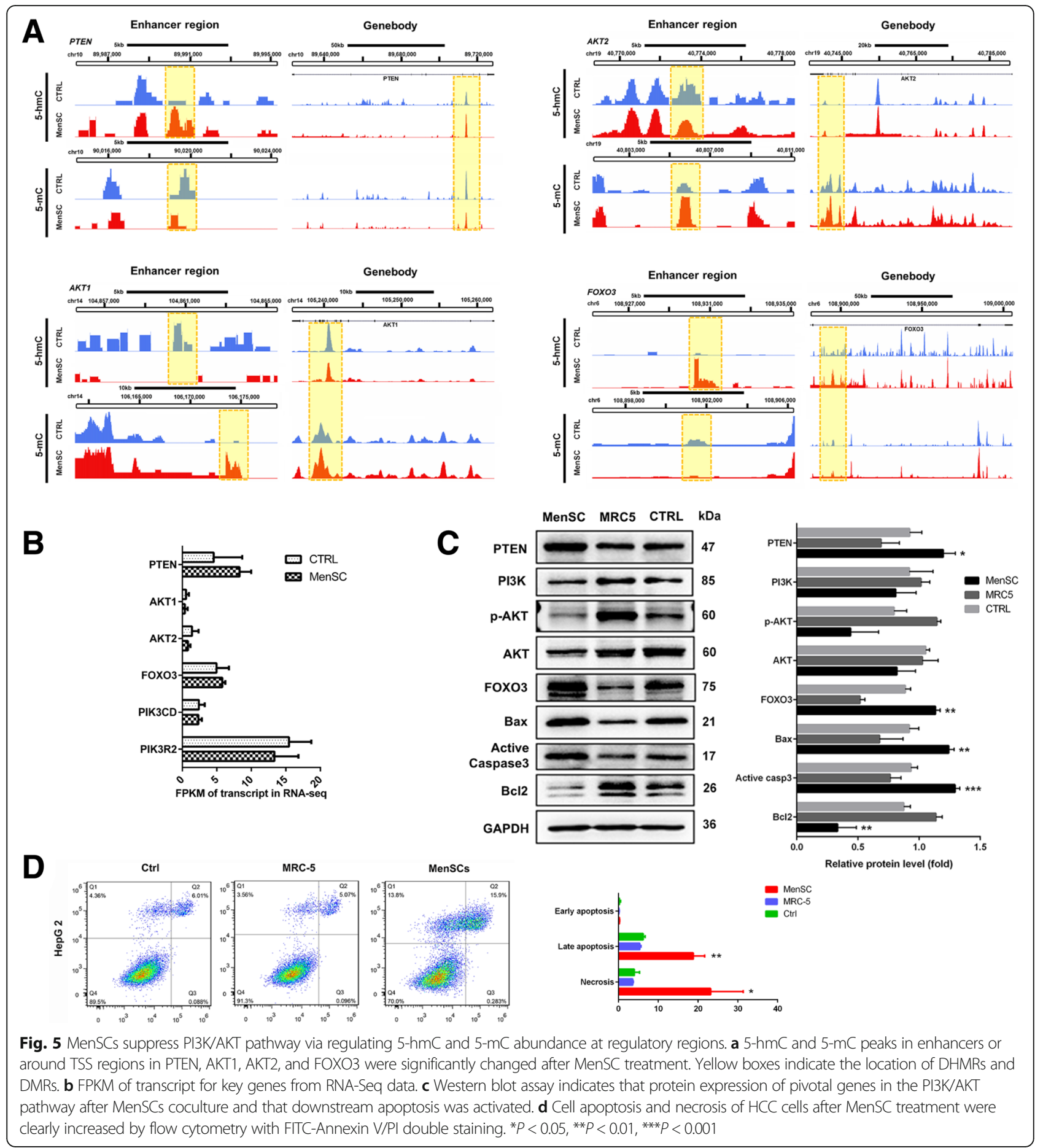

cleaved caspase3 were evidently enhanced, while Bcl-2 was reduced (Fig. 5c). Moreover, flow cytometry assay confirmed that HepG 2 cocultured with MenSCs displayed significantly higher proportions of late apoptotic cells $(18.80 \pm 1.65$ vs $6.19 \pm 0.30, P=0.0017)$ and necrotic cells $(23.17 \pm 4.74$ vs $3.98 \pm 0.78, P=0.0162$, Fig. $5 \mathrm{~d})$. These results indicated that MenSCs promotes apoptosis of HCC through suppression of PI3K/AKT pathway via regulating 5-hmC and 5-mC at enhancers.

\section{MenSCs upregulate E-cadherin via repressing ERK-MYC-} mediated EMT in HCC

The RAF1-ERK pathway plays a vital role in HCC carcinogenesis, progression, and chemotherapy resistance. 
Hence, we focused on whether MenSCs had a suppressive impact on the MAPK pathway through epigenetic alterations. The results showed that MenSCs suppressed the MAPK pathway not only via changing the 5 -hmC and 5-mC levels in the enhancer regions but also through regulating the promoter regions. The $5-\mathrm{hmC}$ level of RAF1 in both the enhancer (chr3:12706241$12706880 ; P=1.97 \mathrm{E}-7)$ and promoter (chr3:12706241$12706880 ; \quad P=1.97 \mathrm{E}-7)$ was significantly decreased under MenSC coculture (Fig. 6a). In addition, 5-hmC levels of MAPK1 (chr22:21607661-21608260; $P=1.10 \mathrm{E}$ -7) and MAPK3 (chr16:29768301-29768540; $P=1.22 \mathrm{E}$ $-5)$ in enhancer regions were clearly decreased.
Moreover, MenSCs also reduced the 5-hmC level in the promoter region of MAPK3 (chr16:30132781-30133160; $P=6.55 \mathrm{E}-6)$. The mRNA expression of MAPK1, MAPK3, and RAF1 correlated with the epigenetic changes (Fig. 6b). Additionally, phosphorylated ERK1/2 in the MenSC group was nearly absent compared with that in the control group (Fig. 6c). C-myc, the downstream target of ERK1/2, was significantly reduced after MenSC coculture. These results indicated that phosphorylation of ERK1/2 was a prerequisite for the activation of c-myc in HCC. Such changes in DNA epigenetic modification probably had a cascading influence on gene expression, including posttranscriptional regulation and

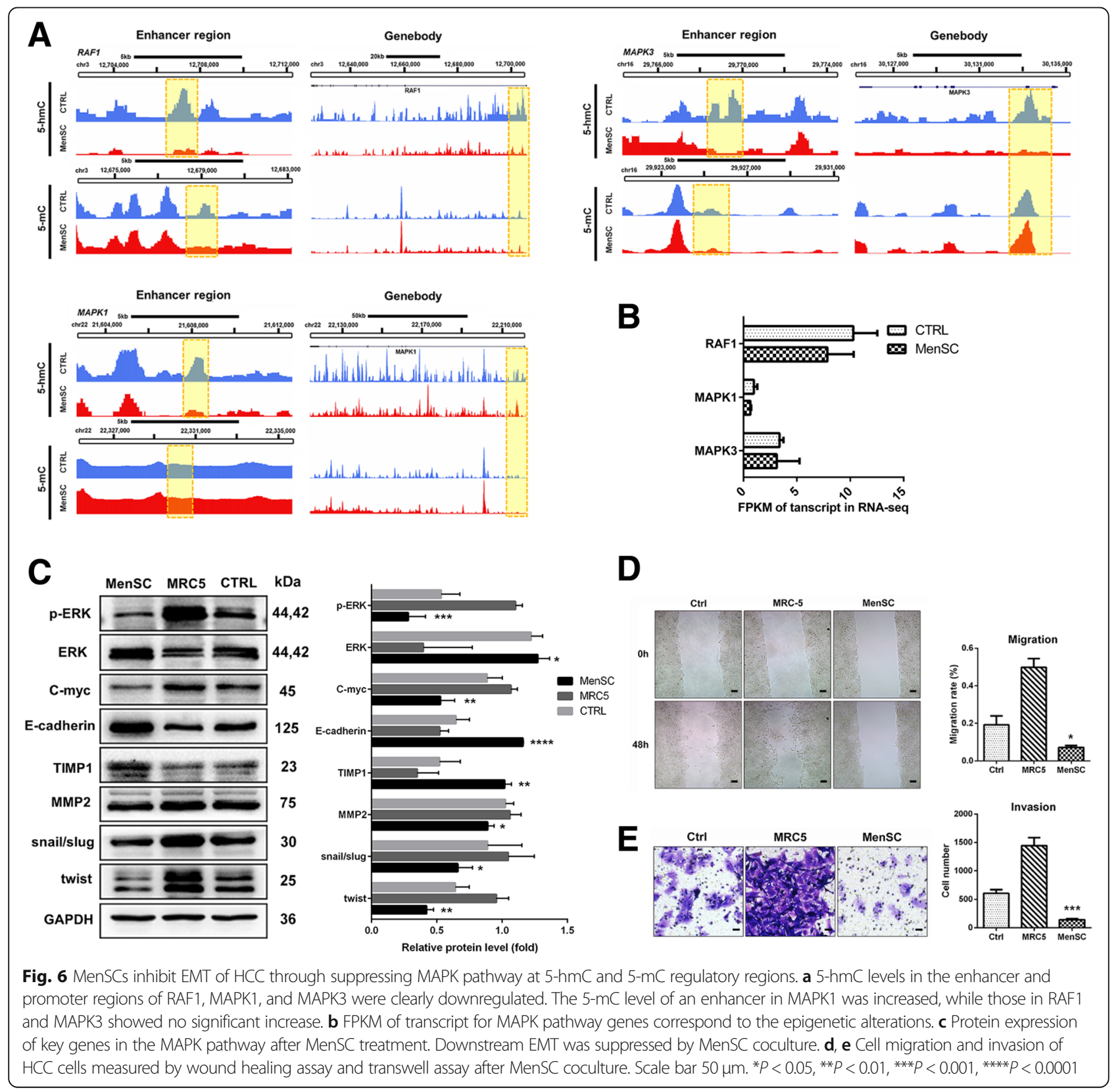


posttranslational modification. Furthermore, we observed that MenSCs could upregulate E-cadherin and TIMP1 and downregulate the MMP2, twist, and snailslug proteins (Fig. 6c). To verify whether MenSCs could inhibit the metastasis and invasion of $\mathrm{HCC}$ in vitro, we conducted a wound healing assay and invasion assay. Compared with control groups, the metastasis and invasion ability of HCC cells was significantly inhibited by MenSCs (Fig. 6d, e). Hence, we speculated that MenSCs hampered the metastasis of HCC cells via suppression of ERK-MYC-EMT axis through epigenetic regulation.

\section{MenSCs downregulated drug resistance genes and} activated tumor suppressors via epigenetic mechanisms

Next, we combined RNA-seq, MeDIP-seq, and hMeDIPseq to identify differentially expressed mRNAs in HCC after MenSC therapy. We observed a clear separation between the MenSC group and the control in the hierarchical clustering analysis of mRNA (Fig. 7a). A total of 266 genes were upregulated, and 123 genes were downregulated in HCC cells with MenSC treatment (Fig. 7b). Based on the integrated analysis, we identified that 206 upregulated genes (206/266, 77.4\%) and 92 downregulated genes (92/123, $74.8 \%$ ) had alterations of 5-hmC and 5-mC at regulatory regions. Among the downregulated genes, we found several drug resistance genes were inhibited via epigenetic regulation (Table 1). Additionally, potential suppressors including LIMA1, NR3C1, IGFBP4, and NDRG1 were upregulated via epigenetic mechanisms (Fig. 7c). Furthermore, ACSS1, HMGA1, and BYSL which closely correlated with tumorigenesis and overall survival were significantly downregulated via 5 -hmC and $5-\mathrm{mC}$ alterations at enhancer and promoter (Fig. 7d, e). Hence, we speculated these genes might be potential targets for gene-modified MSC therapy.

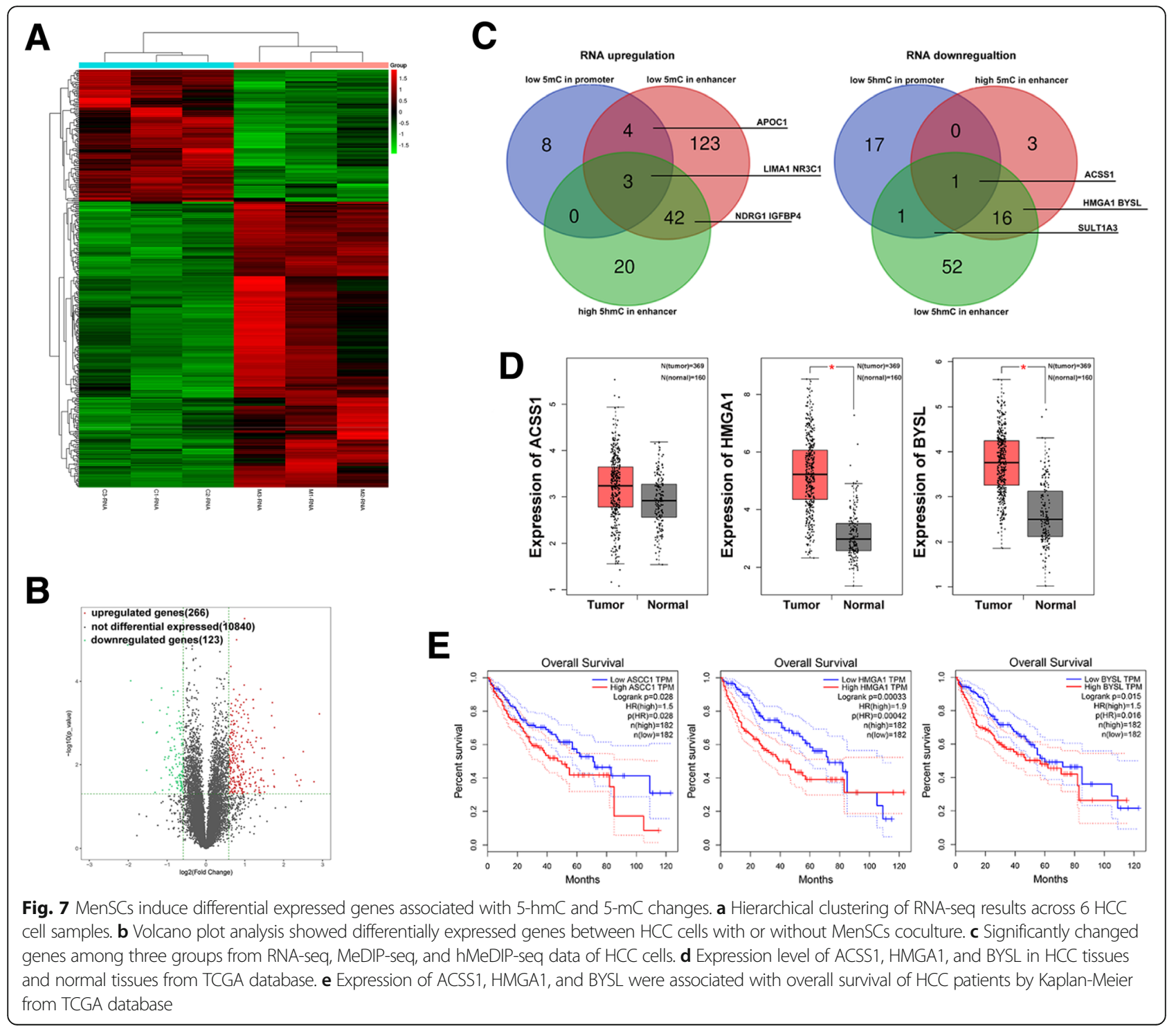


Table 1 Drug resistance-related genes differentially expressed after MenSC coculture via epigenetic alterations

\begin{tabular}{|c|c|c|c|c|}
\hline Genes & $\begin{array}{l}\text { Expression after } \\
\text { MenSC therapy }\end{array}$ & $\begin{array}{l}\text { Epigenetic alteration after } \\
\text { MenSC therapy }\end{array}$ & Expression in literature & Drug resistance \\
\hline ID4 & Downregulated & $\begin{array}{l}5 \mathrm{mC} \text { upregulation in the promoter } \\
5 \text {-hmC downregulation in the enhancer }\end{array}$ & Upregulated & Glioma: 1,3-bis(2-chloroethyl)-1-nitrosourea \\
\hline SQSTM1 & Downregulated & 5-hmC downregulation in the promoter & Upregulated & $\begin{array}{l}\text { Ovarian cancer: cisplatin } \\
\text { Gastric cancer: cisplatin }\end{array}$ \\
\hline HMGA1 & Downregulated & $\begin{array}{l}5 \mathrm{mC} \text { upregulation in the enhancer } \\
5 \text {-hmC downregulation in the enhancer }\end{array}$ & Upregulated & $\begin{array}{l}\text { Lung cancer: tyrosine kinase inhibitor } \\
\text { Ovarian cancer: paclitaxel, doxorubicin } \\
\text { Sarcoma: trabectedin } \\
\text { Cholangiocarcinoma: gemcitabine }\end{array}$ \\
\hline GATA2 & Downregulated & $\begin{array}{l}\text { 5-mC upregulation in the enhancer } \\
5-\mathrm{hmC} \text { downregulation in the enhancer }\end{array}$ & Upregulated & Prostate cancer: docetaxel, cabazitaxel \\
\hline PSAT1 & Downregulated & 5-hmC downregulation in the enhancer & Upregulated & $\begin{array}{l}\text { Colorectal cancer: oxaliplatin } \\
\text { Breast cancer: tamoxifen }\end{array}$ \\
\hline FASN & Downregulated & 5-hmC downregulation in the enhancer & Upregulated & $\begin{array}{l}\text { Ovarian cancer: cisplatin } \\
\text { Lung cancer: tyrosine kinase inhibitor } \\
\text { Gastrointestinal stromal tumor: imatinib } \\
\text { Triple-negative breast cancer: cetuximab }\end{array}$ \\
\hline
\end{tabular}

In addition, MenSCs combined with chemotherapy might be a strategy to alleviate the drug resistance.

\section{Discussion}

The tumor microenvironment plays important roles in tumorigenesis, progression, and drug resistance. Epigenetic mechanisms may function as an interface between environmental factors and the genome, and thus, deregulation of the epigenome by environmental stressors is likely to disrupt a variety of cellular processes [28]. Accumulating evidence indicates that cancer cells not only reshape their epigenomes in response to an altered milieu but also reprogram the tumor microenvironment toward an immunosuppressive state [29]. MSCs are one of the major types of surrounding cell that constitute the tumor microenvironment. Previous studies have confirmed that extraneous MSCs can travel to tumor sites and exert an anticancer effect by altering the microenvironment through multiple paracrine signaling components [30, 31]. However, no reports have indicated whether MSCs can influence the epigenomes of cancer cells via reshaping the microenvironment. Hence, we here presented evidence that MenSCs exert a crucial influence on HCC epigenetics and have an anticancer effect by epigenetically suppressing oncogenic pathways.

In our study, MenSCs could revive the 5-hmC and TET1 expression of HCC cells in a time-dependent manner. TET1, which has been described as a novel suppressor, was depleted, along with $5-\mathrm{hmC}$ reduction, in multiple solid tumors including hepatocarcinoma [32]. Although the mechanism of TET1 reduction in tumorigenesis remains incompletely understood, multiple lines of evidence indicate that TET1 dysregulation plays a major role in the disturbance of DNA demethylation found in solid cancers. TET2 mutations frequently occur in myeloid malignancies and are accompanied by a decline in 5 -hmC and poor prognosis [33]. The mechanism of TET2 inactivation in solid cancer remains elusive, given that TET2 rarely mutates in solid cancer. However, Sajadian et al. [34] found that vitamin $\mathrm{C}$ and 5-azacytidine induce active demethylation, arresting the cell cycle in HCC cells, via TET2. In our study, we also observed an apparent change of TET2 expression in $\mathrm{HuH}-7$ after MenSC treatment. Hence, we speculate MenSCs rescued demethylation of $\mathrm{HCC}$ cells via TET1 or TET2 in different HCC cells. Moreover, MenSCs combined with chemotherapy such as 5-azacytidine might be a potential therapeutic schedule to activate both TET1and TET2-mediated demethylation. The role of TET3 in tumorigenesis is still unclear. Most researchers support the viewpoint that TET1 and probably TET2 are downregulated in solid cancers, while TET3 increases its expression to maintain demethylation for DNA repair and cell survival [35]. In our study, TET3 and DNMT1 were repressed under MenSC coculture. These results indicated that upregulation of TET3 for compensation would not be necessary due to the demethylation mediated by TET1 in HCC that was reactivated by MenSCs. Moreover, DNMT downregulation might be beneficial for tumor suppressor revival via maintaining the balance between DNA methylation and demethylation in genomics.

Previous studies confirmed that MSCs inhibit cancer cells via secretion of diverse components into microenvironment. Yulyana et al. [30] considered that MSC-derived media expressed a high level of insulin growth factor binding proteins (IGFBPs) and can sequester free insulin-like growth factors (IGFs) to inhibit HCC cell proliferation. de Araujo Farias et al. [36] found MSC-derived exosomes enhance radiotherapy-induced cell death in tumor and metastatic tumor foci. In our study, we confirmed that MenSCs have a suppressive effect on HCC cells in vitro 
and vivo through epigenetic regulation via paracrine signaling. Hence, it is indicated that MSCs not only secrete anticancer factors to directly inhibit oncogenic pathways, but also regulate the epigenetic mechanisms of cancer cells to suppress downstream biological functions including apoptosis and metastasis.

Outside of this study, whole-genome hydroxymethylation and methylation profiling have been mainly applied in tumor etiology studies to compare cancer tissues with adjacent tissues. Johnson et al. [37] found that $5-\mathrm{hmC}$ enrichment regions are strongly associated with active transcription in glioblastoma, and 5-hmC was preferentially enriched in glioblastoma-specific enhancers and superenhancers. Additionally, Bhattacharyya et al. [38] revealed that DHMRs were concentrated mostly in regulatory regions and particularly in nonCGIs in pancreatic ductal adenocarcinoma (PDAC). In pancreatic differentiation, DNA hydroxymethylation is positively correlated with enhancer activities and chromatin accessibility [39]. The present study represents the first time that hMeDIP-seq, MeDIP-seq, and RNAseq have been combined to demonstrate the impact of MenSC therapy on epigenetic regulation in HCC cells. We performed genome-wide mapping of the hydroxymethylome and methylome landscapes of HCC cells after MenSC therapy. Most DHMR and DMR localization occurred in the regulatory regions of the genome, such as the enhancer, superenhancer, and promoter regions. These findings demonstrate that dynamic changes in 5-hmC and $5-\mathrm{mC}$ in enhancer regions represent a crucial epigenetic mechanism for regulating gene expression in cell differentiation and tumorigenesis.

PTEN is a major inhibitor of the PI3K/AKT pathway, which is frequently silenced via mutation or promoter hypermethylation in various cancers. Loss of PTEN or activation of PI3K/AKT is involved in cancer progression and drug resistance [40, 41]. However, inhibition of Akt reverses acquired resistance to sorafenib by switching protective autophagy to autophagic cell death in HCC [42]. Lee et al. [43] found that loss of the tumor suppressor IGFBP4, which constitutes an AKT-EZH2 reciprocal loop, drives H3K27me3-mediated epigenetic reprogramming in hepatic carcinogenesis. In the present study, we showed that MenSCs inhibit the PI3K/AKT pathway through regulating 5 -hmC and 5-mC abundance in the enhancer regions of key genes. These results suggested that the redistribution of 5 -hmC abundance mediated by MenSCs in HCC cells is associated with transcriptional activity. Sorafenib is indicated as a treatment for advanced HCC. However, the clinical efficacy of sorafenib is severely compromised by the development of drug resistance. Recently, Kim et al. [44] found that RAF/MEK/ERK signaling was activated by sorafenib and caused sorafenib resistance in HCC. Moreover, phospho-ERK might be a biomarker of response to a synthetic lethal drug combination of sorafenib and MEK inhibition in liver cancer [45]. In the present study, MenSCs suppressed the RAF/ERK/MYC pathway via regulation of $5-\mathrm{hmC}$ and 5-mC levels in enhancer and promoter regions. Furthermore, reductions in phosphorylated ERK1/2 and c-myc repressed downstream EMT. Hence, MAPK pathway would be a vital target for the strategy of modified gene MSC. FOXO3 acts downstream of the PI3K/AKT and RAF/ERK pathways to govern multiple cellular processes including the cell cycle, apoptosis, autophagy, and metabolism. Our study demonstrated that MenSCs could induce the expression of FOXO3 and further promote cell apoptosis in HCC. Fitzwalter et al. [46] found that FOXO3 acts as a cell surveillance mechanism to correct autophagy perturbations and confers apoptosis sensitization if this autophagy imbalance is not rectified.

Demethylation chemotherapeutic drugs, including 5-azacytidine and 5-aza-2'-deoxycytidine, cause wholegenome demethylation and randomly activate the expression of silenced genes. However, we found that MenSCs specifically modulate the expression of target genes through altering 5 -hmC and 5 - $\mathrm{mC}$ abundances in different regions. For instance, MenSCs rescue suppressors, IGFBP4 and NDRG1, via upregulation of 5-hmC and downregulation of 5-mC in enhancer regions, while suppressing ACSS1 expression via downregulation of 5 -hmC in enhancer and promoter regions and upregulating $5-\mathrm{mC}$ in enhancer regions. BYSL protein is upregulated in HCC and required for nucleologenesis in cancer cell proliferation [47]. Overexpression of HMGA1 is significantly associated with tumor progression and EMT in multiple cancers [48, 49]. Suppression of BYSL and HMGA1 might be potential strategy for HCC therapy.

\section{Conclusions}

MenSCs regulated transcriptional activities of PI3K/ $\mathrm{AKT}$ and RAF/ERK via alteration of 5-hmC and 5-mC abundance in gene regulatory regions in $\mathrm{HCC}$ cells. Deactivation of PI3K/AKT and RAF/ERK signaling attenuated the inhibition of FOXO3 and promoted downstream apoptosis. Moreover, an increase in PTEN further strengthened the suppressive effect on PI3K/ AKT. Additionally, inactivation of phosphorylated ERK repressed c-myc-mediated EMT. Taken together, our results provided important epigenetic evidence for clarifying the mechanism of crosstalk between cancer and MSCs in the microenvironment and screening effective epigenetic targets for future therapy strategies based on modified MSCs. 


\section{Additional files}

Additional file 1: Table S1. The sequences of primers for RT-PCR. Table S2. Top 20 pathways of DHMRs in enhancer regions in HCC cells after MenSC coculture. Table S3. Top 20 pathways of DMRs in enhancer regions in HCC cells after MenSC coculture. (XLSX $76 \mathrm{~kb}$ )

Additional file 2: Figure S1. Alterations of 5-hmC and 5-mC of key genes in PI3KVAKT pathway and apoptosis pathway in HCC cells after MenSC treatment. The 5-hmC level of PIK3CD in enhancers was significantly decreased and the 5-mC level in PIK3R2 at enhancers was obviously increased after MenSC coculture. The 5-hmC level in TNFRSF1A and CASP7 at promoters were significantly enhanced. (PDF $1220 \mathrm{~kb}$ )

\section{Abbreviations}

5-hmC: 5-Hydroxymethylcytosine; 5-mC: 5-Methylcytosine;

DHMR: Differentially hydroxymethylated region; DMR: Differentially methylated region; DNMT: DNA methyltransferases; HCC: Hepatocellular carcinoma; hMeDIP-Seq: Hydroxymethylated DNA immunoprecipitation sequencing; MeDIP-Seq: Methylated DNA immune-precipitation sequencing; MenSC: Menstrual blood-derived mesenchymal stem cell; MSC: Mesenchymal stem cell; TET: Tet methylcytosine dioxygenase

\section{Acknowledgements}

Not applicable.

\section{Funding}

This work was supported by grants from the National Key R\&D Program of China (No. 2017YFA0105701).

\section{Availability of data and materials}

All data generated or analyzed during this study are included in this published article. Data of hMeDIP-seq, MeDIP-seq, and RNA-seq in this study have been submitted to the NCBI Gene Expression Omnibus (GEO; https://www.ncbi.nlm. nih.gov/geo) under accession number GSE120160.

\section{Authors' contributions}

YCW and CX designed this research; YCW performed most of the experiment. $X C, Y J Z$, and $Y L W$ participated in in vitro experiments and animal work. YFL analyzed data; YCW wrote the paper and drew the figures. All authors read and approved the final manuscript.

\section{Ethics approval and consent to participate}

Menstrual blood was obtained from volunteers after informed consent. All experimental protocols were approved by The Tab of Animal Experimental Ethical Inspection of the First Affiliated Hospital, College of Medicine, Zhejiang University, approval number 2016-(288).

\section{Consent for publication}

Not applicable.

\section{Competing interests}

The authors declare that they have no competing interests.

\section{Publisher's Note}

Springer Nature remains neutral with regard to jurisdictional claims in published maps and institutional affiliations.

Received: 2 December 2018 Revised: 15 March 2019

Accepted: 24 April 2019 Published online: 31 May 2019

\section{References}

1. Flavahan WA, Gaskell E, Bernstein BE. Epigenetic plasticity and the hallmarks of cancer. Science. 2017;357(6348).

2. Hanahan D, Weinberg RA. Hallmarks of cancer: the next generation. Cell. 2011;144(5):646-74.

3. Okamoto Y, Shinjo K, Shimizu Y, Sano T, Yamao K, Gao W, et al. Hepatitis virus infection affects DNA methylation in mice with humanized livers. Gastroenterology. 2014;146(2):562-72.
4. Sfanos KS, Yegnasubramanian S, Nelson WG, De Marzo AM. The inflammatory microenvironment and microbiome in prostate cancer development. Nat Rev Urol. 2018;15(1):11-24.

5. Qiu GZ, Jin MZ, Dai JX, Sun W, Feng JH, Jin WL. Reprogramming of the tumor in the hypoxic niche: the emerging concept and associated therapeutic strategies. Trends Pharmacol Sci. 2017;38(8):669-86.

6. Barlow DP, Bartolomei MS. Genomic imprinting in mammals. Cold Spring Harb Perspect Biol. 2014;6(2).

7. Hill PWS, Leitch HG, Requena CE, Sun Z, Amouroux R, Roman-Trufero M, et al. Epigenetic reprogramming enables the transition from primordial germ cell to gonocyte. Nature. 2018;555(7696):392-6.

8. Kottakis F, Nicolay BN, Roumane A, Karnik R, Gu H, Nagle JM, et al. LKB1 loss links serine metabolism to DNA methylation and tumorigenesis. Nature. 2016;539(7629):390-5.

9. Gu T, Lin X, Cullen SM, Luo M, Jeong M, Estecio M, et al. DNMT3A and TET1 cooperate to regulate promoter epigenetic landscapes in mouse embryonic stem cells. Genome Biol. 2018;19(1):88.

10. Neumann O, Kesselmeier M, Geffers R, Pellegrino R, Radlwimmer B, Hoffmann $\mathrm{K}$, et al. Methylome analysis and integrative profiling of human HCCs identify novel protumorigenic factors. Hepatology. 2012;56(5):1817-27.

11. Ito S, Shen L, Dai Q, Wu SC, Collins LB, Swenberg JA, et al. Tet proteins can convert 5-methylcytosine to 5-formylcytosine and 5-carboxylcytosine. Science. 2011:333(6047):1300-3

12. Ruzov A, Tsenkina Y, Serio A, Dudnakova T, Fletcher J, Bai Y, et al. Lineagespecific distribution of high levels of genomic 5-hydroxymethylcytosine in mammalian development. Cell Res. 2011;21(9):1332-42.

13. Liu GY, Liu Y, Lu Y, Qin YR, Di GH, Lei YH, et al. Short-term memory of danger signals or environmental stimuli in mesenchymal stem cells: implications for therapeutic potential. Cell Mol Immunol. 2016;13(3):369-78.

14. Jangiam W, Tungjai $M$, Rithidech KN. Induction of chronic oxidative stress, chronic inflammation and aberrant patterns of DNA methylation in the liver of titanium-exposed CBA/CaJ mice. Int J Radiat Biol. 2015;91(5):389-98.

15. Li W, Zhang X, Lu X, You L, Song Y, Luo Z, et al. 5-Hydroxymethylcytosine signatures in circulating cell-free DNA as diagnostic biomarkers for human cancers. Cell Res. 2017;27(10):1243-57.

16. Yang Q, Wu K, Ji M, Jin W, He N, Shi B, et al. Decreased 5hydroxymethylcytosine (5-hmC) is an independent poor prognostic factor in gastric cancer patients. J Biomed Nanotechnol. 2013:9(9):1607-16.

17. Shi Y, Wang Y, Li Q, Liu K, Hou J, Shao C, et al. Immunoregulatory mechanisms of mesenchymal stem and stromal cells in inflammatory diseases. Nat Rev Nephrol. 2018;14(8):493-507.

18. Guiho R, Biteau K, Grisendi G, Taurelle J, Chatelais M, Gantier M, et al. TRAlL delivered by mesenchymal stromal/stem cells counteracts tumor development in orthotopic Ewing sarcoma models. Int J Cancer. 2016; 139(12):2802-11.

19. Jeong KY, Lee EJ, Kim SJ, Yang SH, Sung YC, Seong J. Irradiation-induced localization of IL-12-expressing mesenchymal stem cells to enhance the curative effect in murine metastatic hepatoma. Int J Cancer. 2015;137(3):721-30.

20. Ong HT, Federspiel MJ, Guo CM, Ooi LL, Russell SJ, Peng KW, et al. Systemically delivered measles virus-infected mesenchymal stem cells can evade host immunity to inhibit liver cancer growth. J Hepatol. 2013;59(5):999-1006.

21. Rossignoli F, Caselli A, Grisendi G, Piccinno S, Burns JS, Murgia A, et al. Isolation, characterization, and transduction of endometrial decidual tissue multipotent mesenchymal stromal/stem cells from menstrual blood. Biomed Res Int. 2013:2013:901821.

22. Zhong Z, Patel AN, Ichim TE, Riordan NH, Wang H, Min WP, et al. Feasibility investigation of allogeneic endometrial regenerative cells. J Transl Med. 2009;7:15.

23. Chen L, Zhang C, Chen L, Wang X, Xiang B, Wu X, et al. Human menstrual blood-derived stem cells ameliorate liver fibrosis in mice by targeting hepatic stellate cells via paracrine mediators. Stem Cells Transl Med. 2017; 6(1):272-84

24. Chen $L$, Xiang $B$, Wang $X$, Xiang $C$. Exosomes derived from human menstrual blood-derived stem cells alleviate fulminant hepatic failure. Stem Cell Res Ther. 2017:8(1):9.

25. Xiang B, Chen L, Wang X, Zhao Y, Wang Y, Xiang C. Transplantation of menstrual blood-derived mesenchymal stem cells promotes the repair of LPS-induced acute lung injury. Int J Mol Sci. 2017;18(4). https://doi.org/10. 3390/ijms18040689.

26. Bu S, Wang Q, Zhang Q, Sun J, He B, Xiang C, et al. Human endometrial mesenchymal stem cells exhibit intrinsic anti-tumor properties on human epithelial ovarian cancer cells. Sci Rep. 2016;6:37019. 
27. Wu X, Luo Y, Chen J, Pan R, Xiang B, Du X, et al. Transplantation of human menstrual blood progenitor cells improves hyperglycemia by promoting endogenous progenitor differentiation in type 1 diabetic mice. Stem Cells Dev. 2014:23(11):1245-57.

28. Herceg Z, Vaissiere T. Epigenetic mechanisms and cancer: an interface between the environment and the genome. Epigenetics. 2011;6(7):804-19.

29. Liu M, Zhou J, Chen Z, Cheng AS. Understanding the epigenetic regulation of tumours and their microenvironments: opportunities and problems for epigenetic therapy. J Pathol. 2017;241(1):10-24.

30. Yulyana Y, Ho IA, Sia KC, Newman JP, Toh XY, Endaya BB, et al. Paracrine factors of human fetal MSCs inhibit liver cancer growth through reduced activation of IGF1R/PIIK/Akt signaling. Mol Ther. 2015;23(4):746-56.

31. Bliss SA, Sinha G, Sandiford OA, Williams LM, Engelberth DJ, Guiro K, et al. Mesenchymal stem cell-derived exosomes stimulate cycling quiescence and early breast cancer dormancy in bone marrow. Cancer Res. 2016;76(19):5832-44.

32. Chen $Q$, Yin D, Zhang Y, Yu L, Li XD, Zhou Z, et al. MicroRNA-29a induces loss of 5-hydroxymethylcytosine and promotes metastasis of hepatocellular carcinoma through a TET-SOCS1-MMP9 signaling axis. Cell Death Dis. 2017;8(6):e2906.

33. Ko M, Huang Y, Jankowska AM, Pape UJ, Tahiliani M, Bandukwala HS, et al. Impaired hydroxylation of 5-methylcytosine in myeloid cancers with mutant TET2. Nature. 2010;468(7325):839-43.

34. Sajadian SO, Tripura C, Samani FS, Ruoss M, Dooley S, Baharvand H, et al. Vitamin C enhances epigenetic modifications induced by 5-azacytidine and cell cycle arrest in the hepatocellular carcinoma cell lines HLE and Huh7. Clin Epigenetics. 2016;8:46.

35. Kantidze OL, Razin SV. 5-Hydroxymethylcytosine in DNA repair: a new player or a red herring? Cell Cycle. 2017:16(16):1499-501.

36. de Araujo Farias V, OValle F, Serrano-Saenz S, Anderson P, Andres E, LopezPenalver J, et al. Exosomes derived from mesenchymal stem cells enhance radiotherapy-induced cell death in tumor and metastatic tumor foci. Mol Cancer. 2018;17(1):122.

37. Johnson KC, Houseman EA, King JE, von Herrmann KM, Fadul CE, Christensen BC 5-Hydroxymethylcytosine localizes to enhancer elements and is associated with survival in glioblastoma patients. Nat Commun. 2016;7:13177.

38. Bhattacharyya S, Pradhan K, Campbell N, Mazdo J, Vasantkumar A, Maqbool S, et al. Altered hydroxymethylation is seen at regulatory regions in pancreatic cancer and regulates oncogenic pathways. Genome Res. 2017;27(11):1830-42.

39. Li J, Wu X, Zhou Y, Lee M, Guo L, Han W, et al. Decoding the dynamic DNA methylation and hydroxymethylation landscapes in endodermal lineage intermediates during pancreatic differentiation of hESC. Nucleic Acids Res. 2018; 46(6):2883-900.

40. Lee MS, Jeong MH, Lee HW, Han HJ, Ko A, Hewitt SM, et al. PISK/AKT activation induces PTEN ubiquitination and destabilization accelerating tumourigenesis. Nat Commun. 2015;6:7769.

41. Mills JN, Rutkovsky AC, Giordano A. Mechanisms of resistance in estrogen receptor positive breast cancer: overcoming resistance to tamoxifen/aromatase inhibitors. Curr Opin Pharmacol. 2018;41:59-65.

42. Zhai B, Hu F, Jiang $X, X u J$, Zhao D, Liu B, et al. Inhibition of Akt reverses the acquired resistance to sorafenib by switching protective autophagy to autophagic cell death in hepatocellular carcinoma. Mol Cancer Ther. 2014;13(6):1589-98.

43. Lee YY, Mok MT, Kang W, Yang W, Tang W, Wu F, et al. Loss of tumor suppressor IGFBP4 drives epigenetic reprogramming in hepatic carcinogenesis. Nucleic Acids Res. 2018;46(17):8832-47.

44. Kim JS, Choi GH, Jung Y, Kim KM, Jang SJ, Yu ES, et al. Downregulation of Raf-1 kinase inhibitory protein as a sorafenib resistance mechanism in hepatocellular carcinoma cell lines. J Cancer Res Clin Oncol. 2018;144(8):1487-501.

45. Wang C, Jin H, Gao D, Lieftink C, Evers B, Jin G, et al. Phospho-ERK is a biomarker of response to a synthetic lethal drug combination of sorafenib and MEK inhibition in liver cancer. J Hepatol. 2018;69(5):1057-65.

46. Fitzwalter BE, Thorburn A. FOXO3 links autophagy to apoptosis. Autophagy. 2018 14(8):1467-8

47. Wang H, Xiao W, Zhou Q, Chen Y, Yang S, Sheng J, et al. Bystin-like protein is upregulated in hepatocellular carcinoma and required for nucleologenesis in cancer cell proliferation. Cell Res. 2009;19(10):1150-64.

48. Andreozzi M, Quintavalle C, Benz D, Quagliata L, Matter M, Calabrese D, et al. HMGA1 expression in human hepatocellular carcinoma correlates with poor prognosis and promotes tumor growth and migration in in vitro models. Neoplasia. 2016;18(12):724-31.

49. Liang L, Li X, Zhang X, Lv Z, He G, Zhao W, et al. MicroRNA-137, an HMGA1 target, suppresses colorectal cancer cell invasion and metastasis in mice by directly targeting FMNL2. Gastroenterology. 2013;144(3):624-35 e4.

Ready to submit your research? Choose BMC and benefit from:

- fast, convenient online submission

- thorough peer review by experienced researchers in your field

- rapid publication on acceptance

- support for research data, including large and complex data types

- gold Open Access which fosters wider collaboration and increased citations

- maximum visibility for your research: over $100 \mathrm{M}$ website views per year

At BMC, research is always in progress.

Learn more biomedcentral.com/submissions 